\title{
An exact solution of three interacting friendly walks in the bulk $\ddagger$
}

\author{
R Tabbara ${ }^{1}$ and A L Owczarek ${ }^{1}$ and A Rechnitzer ${ }^{2}$ \\ ${ }^{1}$ School of Mathematics and Statistics, The University of Melbourne, Victoria 3010, \\ Australia. \\ ${ }^{2}$ Department of Mathematics, University of British Columbia, Vancouver V6T 1Z2, \\ British Columbia, Canada. \\ E-mail: tabbarar@ms.unimelb.edu.au, owczarek@unimelb.edu.au, \\ andrewr@math . ubc .ca
}

\begin{abstract}
We find the exact solution of three interacting friendly directed walks on the square lattice in the bulk, modelling a system of homopolymers that can undergo gelation by introducing two distinct interaction parameters that differentiate between the zipping of only two or all three walks. We establish functional equations for the model's corresponding generating function that are subsequently solved exactly by means of the obstinate kernel method. We then proceed to analyse our model, first considering the case where triple-walk interaction effects are ignored, finding that our model exhibits two phases which we classify as free and gelated regions, with the system exhibiting a second-order phase transition. We then analyse the full model where both interaction parameters are incorporated, presenting the full phase diagram and highlighting the additional existence of a first-order gelation boundary.
\end{abstract}

PACS numbers: 05.50.+q, 05.70.fh, 61.41.+e

Submitted to: J. Phys. A.: Math. Theor.

$\ddagger$ Dedicated to Professor Rodney Baxter on the occasion of his 75th birthday 


\section{Introduction}

To model the phase behaviour of polymer gelation requires the consideration of systems of multiple polymers [1]. The study of two polymers with interpolymer interactions and interacting with a surface has received much recent attention because of connections to modelling the unzipping of DNA. Typically these have been modelled via either selfavoiding or directed walk systems on lattices in two and three dimensions with various types of contact interactions [2-10]. The exact solution of directed friendly walkers on the square lattice with such interactions [9, 10] has led to the extension of a key combinatorial technique for lattice paths, the obstinate kernel method [11].

To extend these integrable models to the problem of polymer gelation we solve a system of three polymer strands with contact interactions. We model this system by an ensemble of three directed friendly walkers with shared-vertex interactions on the square lattice. We introduce two different types of contact interactions that differentiate between situations in which two walks share the same site or all three walks share the same site.

We begin in Section 2 by constructing our model, first defining the combinatorial class of allowed configurations and subsequently introducing interaction parameters to assign our configurations with corresponding Boltzmann weights. In particular, we incorporate two distinct interaction parameters to differentiate between the zipping of only two or all three walks.

In Section 3, we introduce two further variables that mark the final vertical distances between our three walks for any given configuration. These auxiliary variables, known as catalytic variables, are integral to solving our model. We then establish a mapping between our class of allowed triple-walks onto itself which leads to a functional equation for the model's corresponding generating function that incorporates our added parameters.

We then proceed in Section 4 to determine an exact solution to the model's generating function by means of the obstinate kernel method [11]. While the beginnings of Section 4 outline the precise steps undertaken, we briefly mention that this technique consists of generating a finite system of distinct functional equations by applying a set of different transformations to our original relation determined in Section 3 , We then subsequently collapse our system to construct a new refined functional equation which provides us (after some further work) with a solution to our generating function.

Equipped with our exact solution of the generating function, we proceed to analyse our model in Section 5. Specifically, Section 5.1 first considers the dominant singularity behaviour of the generating function for the simplified model where we ignore triple contact effects. We find that such a model exhibits a single critical point, arising in two distinct phases of our system - namely, a free and gelated phase. In Section 5.2 we determine that the free to gelated phase transition is a second-order, with a finite jump discontinuity in the second-derivative of free energy.

In Section 5.3 we extend our analysis to the full symmetric model that incorporates 
both double and triple interaction effects, specifying the regions of the phases and plotting the phase diagram. While the full phase space is similarly partitioned into two distinct phases, we find the existence of an additional first-order phase boundary for relatively low double and high triple interaction Boltzmann weights.

Finally, in Section 5.4, by a simple re-parameterisation, we consider the model that purely isolates double and triple walker interaction effects. We plot the new phase diagram and use low and high-temperature arguments to explain the limiting behaviour of our phase boundaries. As one might expect, the order of all phase transitions across the entire phase space remain unchanged.

\section{The model}

Consider three directed walks along the square integer lattice consisting of an equal number of steps. All walks begin at the origin and end at the same site. Moreover, walks only can take steps in either the north-east $(1,1)$ or south-east $(1,-1)$ direction. Finally, all three walks may share common steps, however none of the walks are able to cross one another. Such walks are typically referred to as (infinitely) friendly walks. Let $\widehat{\Omega}$ denote the class of allowed triple walks of any length. An example of an allowable configuration is given in Figure 1.

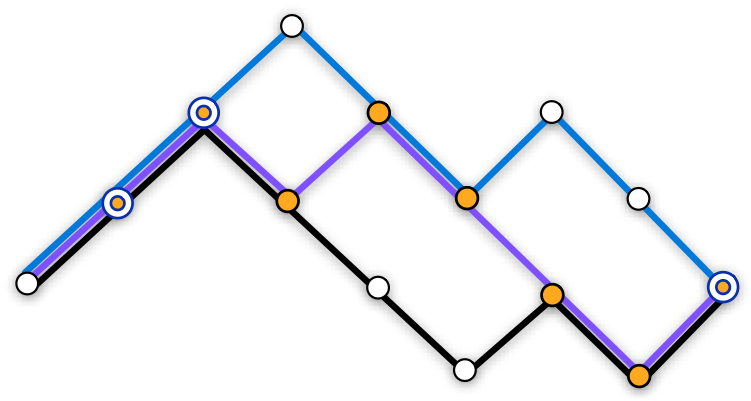

Figure 1. An example of an allowed configuration of length 8 . Here, we have $m_{c}=11$ double shared contact steps and $m_{d}=3$ triple shared contact sites. Thus, the overall Boltzmann weight for this configuration is $c^{11} d^{3}$

For any configuration $\varphi \in \widehat{\Omega}$, we assign a weight $c$ to the $m_{c}(\varphi)$ shared contact sites between the top-to-middle or middle-to-bottom walks respectively. Note, that when all three walks share the same site we consider the walk as consisting of two shared contacts sites with corresponding weight $c^{2}$ and further the trivial triplet of walks of zero length has weight 1 . Finally, we assign a weight $d$ to the $m_{d}(\varphi)$ triple shared contact sites where all three walks coincide, hence contributing a total factor of $c^{2} d$ to the overall configuration weight. The partition function for our model consisting of $n$ paired steps 
is

$$
Z_{n}(c, d)=\sum_{\varphi \in \widehat{\Omega},|\varphi|=n} c^{m_{c}(\varphi)} d^{m_{d}(\varphi)},
$$

where $|\varphi|$ denotes the length of the configuration $\varphi$. The reduced free energy $\psi(c, d)$

$$
\psi(c, d)=-\lim _{n \rightarrow \infty} \frac{1}{n} \log Z_{n}(c, d) .
$$

and generating function $G(c, d ; z)$

$$
G(c, d ; z)=\sum_{n=0}^{\infty} Z_{n}(c, d) z^{n}
$$

are defined in the usual manner, where $z$ is conjugate to the length of the configuration.

Let $\widehat{\Omega}_{P}$ be the subclass of allowed configurations where all three walks share a common site only at the very beginning and end of the configuration. An example of such a configuration is seen in Figure 2. We can then define the corresponding primitive

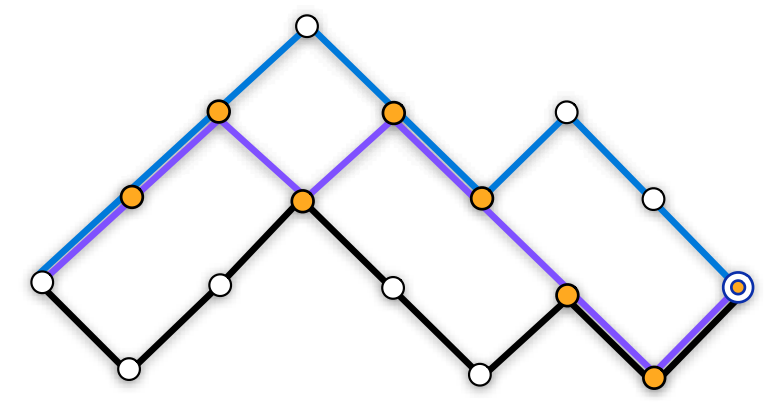

Figure 2. An example of a primitive configuration where all three walks only coincide on the first and last visited sites.

generating function $P(c ; z)$ as

$$
P(c ; z)=\sum_{\varphi \in \widehat{\Omega}_{P}} z^{|\varphi|} c^{m_{c}(\varphi)}
$$

where $z$ is conjugate to the length $|\varphi|$ of a configuration $\varphi \in \widehat{\Omega}_{P}$. Note that we can append any two configurations, $\varphi_{1}, \varphi_{2} \in \widehat{\Omega}_{P}$ together to construct a new configuration $\varphi=\varphi_{1} \cdot \varphi_{2}$ that now lies in our original class $\widehat{\Omega}$. More generally any $\varphi \in \widehat{\Omega}$ can be uniquely decomposed into a sequence of primitive walks appended to each other as highlighted in Figure 2. Using the symbolic enumeration formalism described by Flajolet and Sedgewick [12] we have

$$
\widehat{\Omega}=S E Q\left(\widehat{\Omega}_{P}\right)=\{\bullet\}+\widehat{\Omega}_{P}+\left\{\widehat{\Omega}_{P} \times \widehat{\Omega}_{P}\right\}+\left\{\widehat{\Omega}_{P} \times \widehat{\Omega}_{P} \times \widehat{\Omega}_{P}\right\}+\ldots
$$

where $\{\bullet\}$ denotes the trivial configuration consisting of no steps and

$$
\left\{\prod_{i=1}^{N} \widehat{\Omega}_{P}\right\}=\left\{\varphi_{1} \cdot \varphi_{2} \cdot \ldots \varphi_{N} \mid \varphi_{i} \in \widehat{\Omega}_{P}\right\} .
$$



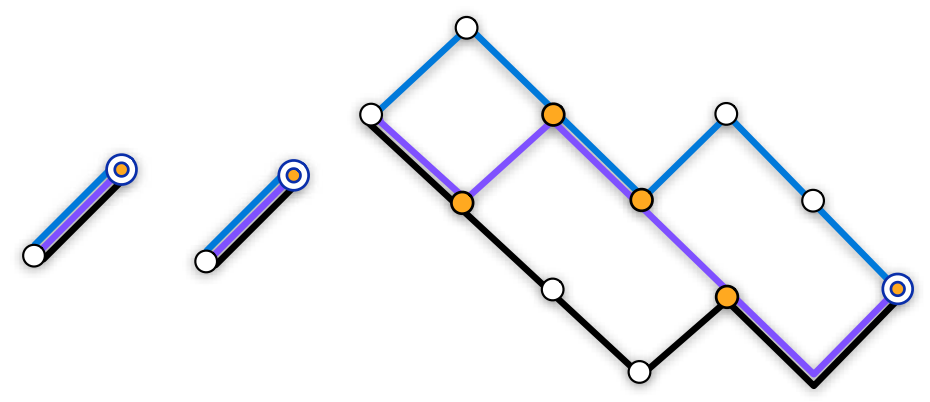

Figure 3. Decomposing the configuration seen in Figure 1 into its primitive components

At the level of generating functions, this construction translates to the following equation

$$
\begin{aligned}
G(c, d ; z) & =1+d P(c ; z)+d^{2} P(c ; z)^{2}+\ldots \\
& =\frac{1}{1-d P(c ; z)} .
\end{aligned}
$$

Letting $d=1$ we can express our primitive generating function in terms of $G(c, 1 ; z)$

$$
P(c ; z)=\frac{G(c, 1 ; z)-1}{G(c, 1 ; z)},
$$

which further gives us the relation

$$
G(c, d ; z)=\frac{G(c, 1 ; z)}{d[1-G(c, 1 ; z)]+G(c, 1 ; z)} .
$$

Hence to solve for our full model it suffices to solve for the model that ignores triple shared contact effects with corresponding generating function $G(c, 1 ; z)$ which we will indeed proceed to do in Section 3 and Section 4 .

\section{Constructing the functional equations}

We can establish a functional equation for $G(c, 1 ; z)$ by considering the effect of appending a triplet of steps to the end of any given configuration $\varphi \in \widehat{\Omega}$. To begin, we define $\Omega(i, j)$ to be the class of triple walks that consists of configurations with final top to middle walk distance $i$ and middle to bottom distance $j$, that still obey friendly constraints. We define our larger combinatorial class $\Omega\left(0^{+}, 0^{+}\right)$as

$$
\Omega\left(0^{+}, 0^{+}\right) \equiv \bigcup_{i \geq 0, j \geq 0} \Omega(i, j)
$$

Note that our original class of walks in our model $\widehat{\Omega} \equiv \Omega(0,0)$. Equipped with our larger combinatorial class we can introduce its corresponding generating function $F(c ; z)$ that encodes information about the number of steps and shared contacts for each configuration $\varphi \in \Omega\left(0^{+}, 0^{+}\right)$. However, determining whether appending a triple-step 
onto a given configuration $\varphi$ results in a new and allowable configuration (i.e. $\varphi$ remains in $\Omega\left(0^{+}, 0^{+}\right)$) further requires knowledge of the final step distances between the three walks. Hence, solely for the purpose of establishing our functional equation for $F(c ; z)$, we additionally introduce two catalytic variables $r$ and $s$ to construct the expanded generating function $F(r, s, c ; z)$ where

$$
F(r, s, c ; z) \equiv F(r, s)=\sum_{\varphi \in \Omega\left(0^{+}, 0^{+}\right)} z^{|\varphi|} r^{h(\varphi) / 2} s^{f(\varphi) / 2} c^{m_{c}(\varphi)}
$$

and again $z$ is conjugate to the length $|\varphi|$ of a configuration $\varphi \in \Omega\left(0^{+}, 0^{+}\right), r$ and $s$ are conjugate to half the distance $h(\varphi)$ and $f(\varphi)$ between the final vertices of the top to middle and middle to bottom walks respectively. For each $\varphi \in \Omega\left(0^{+}, 0^{+}\right)$, powers of $r$ and $s$ in $F(r, s)$ track the final step distances between the three walks. Due to the allowed step directions, both $h(\varphi)$ and $f(\varphi)$ must always be even, ensuring that $F(r, s)$ contains only integer powers of $r$ and $s$. Thus, we consider $F(r, s)$ as an element of $\mathbb{Z}[r, s, c][[z]]$ : the ring of formal power series in $z$ with coefficients in $\mathbb{Z}[r, s, c]$.

We aim to solve $F(0,0, c ; z) \equiv G(c, 1 ; z)$ by establishing a functional equation for $F(r, s)$. Specifically, we construct a suitable mapping from $\Omega\left(0^{+}, 0^{+}\right)$onto itself by considering the effect of appending an allowable triple-step onto a configuration, translating this map into its action on the generating function. At the end of any given walk we can append a step $(1, \pm 1)$. Hence, for a triplet of walks, there are a total of eight possible combinations of triple steps that can be appended onto a configuration. Let $\mathcal{S}$ be the set of allowable steps, with

$\mathcal{S}=\{(1,1,1),(-1,1,1),(1,-1,1),(1,1,-1),(-1,-1,1),(-1,1,-1),(1,-1,-1),(-1,-1,-1)\}$

that alter the corresponding configuration weight by factors of $z, \frac{z}{r}, \frac{z r}{s}, z s, \frac{z}{s}, \frac{z s}{r}$, $z r$ and $z$ respectively. Note, in (12) we have used the shorthand $(x, y, z)$ to denote the triple-step $\{(1, x),(1, y),(1, z)\}$.

Given the non-crossing constraint between the three walks, not all eight combinations of appended steps will necessarily result in allowable configurations and furthermore shared contact interaction effects also need to be considered when attaching new steps. Thus, we identify 22 distinct cases that capture all possible changes in weight that can arise from appending a triplet of steps as seen in Figure 4, Figure 5 and Figure 6 , allowing us to construct a functional equation for $F(r, s)$, highlighting the underlying decomposition for $\Omega\left(0^{+}, 0^{+}\right)$. We denote $\{\bullet\}$ as the trivial zero-length configuration and introduce the following shorthand notation

$$
\Omega\left(n^{+}, j\right) \equiv \bigcup_{i \geq n} \Omega(i, j), \quad \Omega\left(i, m^{+}\right) \equiv \bigcup_{j \geq m} \Omega(i, j), \quad \Omega\left(n^{+}, m^{+}\right)=\bigcup_{i \geq n, j \geq m} \Omega(i, j)
$$

while

$$
\{\sigma\} \cdot \Omega(i, j)
$$

represents the class of configurations formed by appending the triple-step $\sigma \in \mathcal{S}$ to the end of each triplet of walks $\varphi \in \Omega(i, j)$. We can build up our functional equation 


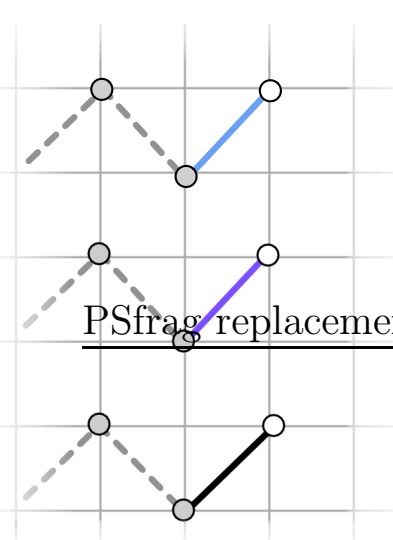

(a)

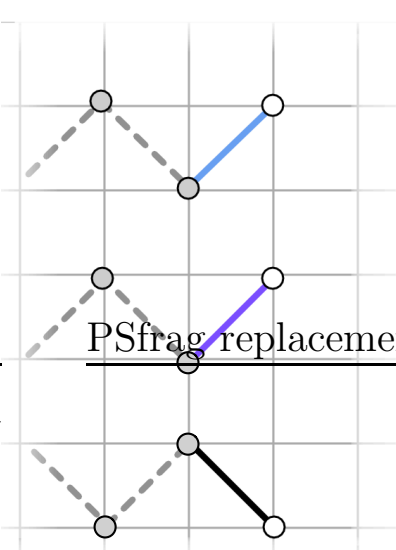

(d)

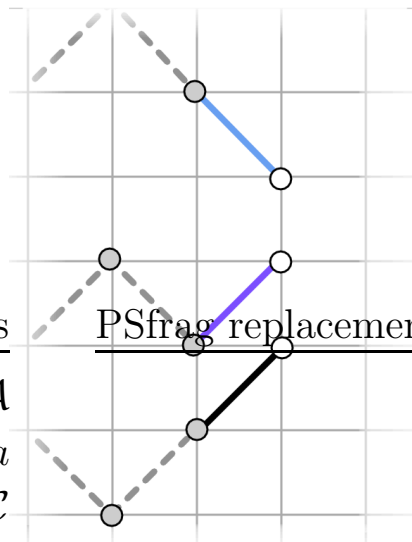

(b)

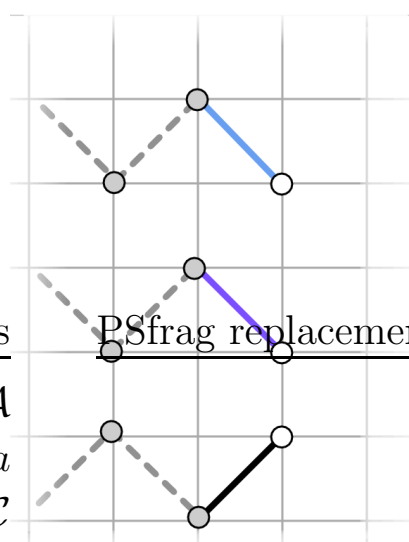

(e)

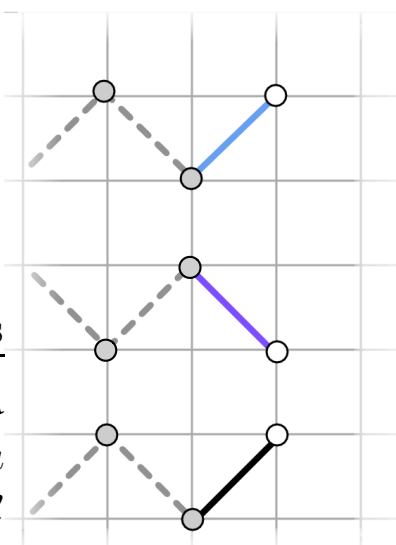

(c)

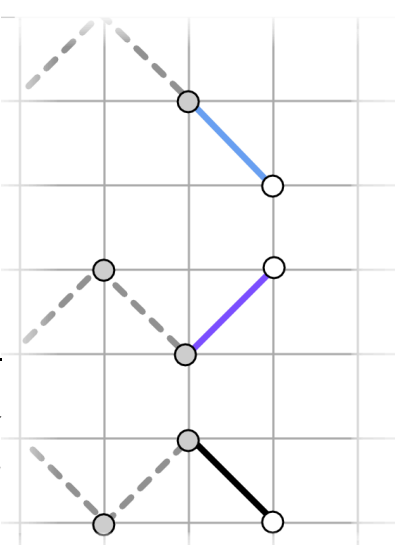

(f)

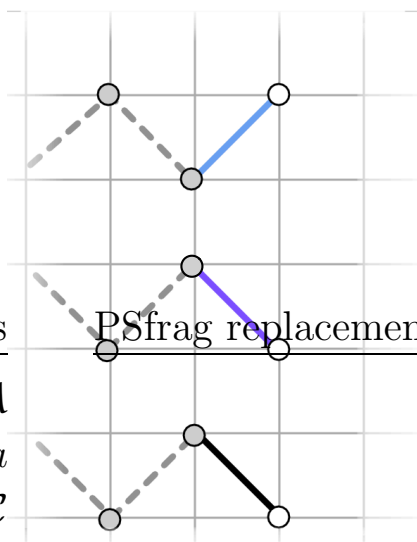

(g)

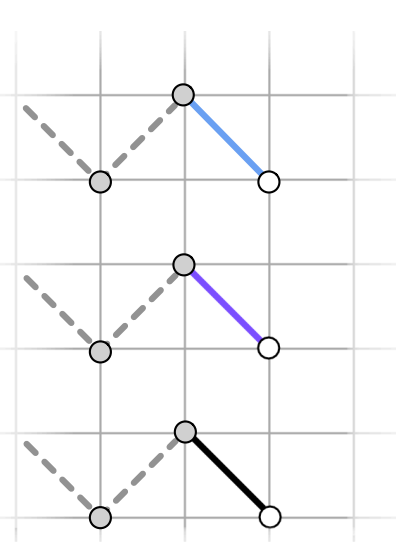

(h)

Figure 4. The eight possible ways of appending a triplet of steps to an allowed configuration that results in no new shared contacts. 
$F(r, s, c ; z)$ by firstly establishing a relation for the non-interacting case $F(r, s, 1 ; z)$ and subsequently incorporating the effects of shared contacts. To do this we consider the effect of appending a triple-step onto a given configuration, making sure to eliminate newly formed walks that are no longer part of our allowable class $\Omega\left(0^{+}, 0^{+}\right)$. For $F(r, s, 1 ; z)$ we find

$$
\begin{array}{lll}
F(r, s, 1,1 ; z)= & \Omega\left(0^{+}, 0^{+}\right)= \\
1 & \{\bullet\} & \\
+z F(r, s) & \bigcup\{(1,1,1)\} \cdot \Omega\left(0^{+}, 0^{+}\right), & \text {Figure 4a } \\
+\frac{z}{r}\left(F(r, s)-\left[r^{0}\right] F(r, s)\right) & \bigcup\{(-1,1,1)\} \cdot \Omega\left(1^{+}, 0^{+}\right), & \text {Figure 4b } \\
+\frac{z r}{s}\left(F(r, s)-\left[s^{0}\right] F(r, s)\right) & \bigcup\{(1,-1,1)\} \cdot \Omega\left(0^{+}, 1^{+}\right), & \text {Figure 4c } \\
+z s F(r, s) & \bigcup\{(1,1,-1)\} \cdot \Omega\left(0^{+}, 0^{+}\right), & \text {Figure 4d] } \\
+\frac{z}{s}\left(F(r, s)-\left[s^{0}\right] F(r, s)\right) & \bigcup\{(-1,-1,1)\} \cdot \Omega\left(0^{+}, 1^{+}\right), & \text {Figure 4e } \\
+\frac{z s}{r}\left(F(r, s)-\left[r^{0}\right] F(r, s)\right) & \bigcup\{(-1,1,-1)\} \cdot \Omega\left(1^{+}, 0^{+}\right), & \text {Figure 4f } \\
+z r F(r, s) & \bigcup\{(1,-1,-1)\} \cdot \Omega\left(0^{+}, 0^{+}\right), & \text {Figure 4g } \\
+z F(r, s) & \bigcup\{(-1,-1,-1)\} \cdot \Omega\left(0^{+}, 0^{+}\right), & \text {Figure 4h, }
\end{array}
$$

where $\left[r^{j}\right] F(r, s),\left[s^{k}\right] F(r, s)$ and in general $\left[r^{j} s^{k}\right] F(r, s)$ denote the coefficients of $r^{j}, s^{k}$ and $r^{j} s^{k}$ in the generating function $F(r, s)$ respectively. Note, that since the coefficients of $F(r, s)$ are polynomials in $r, s$ we have

$$
\begin{aligned}
& {\left[r^{0}\right] F(r, s)=F(0, s)} \\
& {\left[s^{0}\right] F(r, s)=F(r, 0),} \\
& {\left[r^{0} s^{0}\right] F(r, s)=F(0,0) .}
\end{aligned}
$$


Next, we add shared contact site effects to (15) to get a functional equation for $F(r, s, c ; z)$, with

$$
\begin{aligned}
& F(r, s, c, 1 ; z)=\text { RHS of (15) } \\
& +z\left(c^{2}-1\right) F(0,0) \\
& \bigcup\{(1,1,1)\} \cdot \Omega(0,0), \quad \text { Figure 5a } \\
& +z\left(c^{2}-1\right) F(0,0) \\
& +z\left(c^{2}-1\right)\left[s^{1}\right] F(0, s) \\
& \bigcup\{(-1,-1,-1)\} \cdot \Omega(0,0), \quad \text { Figure } 5 \mathrm{~b} \\
& +z\left(c^{2}-1\right)\left[r^{1}\right] F(r, 0) \\
& \bigcup\{(-1,-1,1)\} \cdot \Omega(0,2), \quad \text { Figure } 5 \mathrm{c} \\
& +z(c-1) F(0, s) \\
& +z(c-1)(F(0, s)-F(0,0)) \\
& +z(c-1)(F(0, s)-F(0,0)) \\
& +\frac{z}{s}(c-1)\left(F(0, s)-F(0,0)-s\left[s^{1}\right] F(0, s)\right) \\
& \bigcup\{(-1,1,1)\} \cdot \Omega(2,0), \quad \text { Figure } 5 \mathrm{~d} \\
& \bigcup\{(1,1,-1)\} \cdot \Omega\left(0,0^{+}\right), \quad \text { Figure 6a } \\
& +z(c-1)\left(\left[s^{1}\right] F(r, s)-\left[s^{1}\right] F(0, s)\right) \\
& \bigcup\{(1,1,1)\} \cdot \Omega\left(0,2^{+}\right), \quad \text { Figure } 6 \mathrm{~b} \\
& \bigcup\{-1,-1,-1\} \cdot \Omega\left(0,2^{+}\right), \quad \text { Figure } 6 \mathrm{c} \\
& +\frac{z}{r}(c-1)\left(F(r, 0)-F(0,0)-r\left[r^{1}\right] F(r, 0)\right) \\
& \bigcup\{-1,-1,1\} \cdot \Omega\left(0,4^{+}\right), \quad \text { Figure 6d } \\
& \bigcup\{(-1,-1,1)\} \cdot \Omega\left(2^{+}, 2\right), \quad \text { Figure } 6 \mathrm{e} \\
& +z r(c-1)(F(r, 0)-F(0,0)) \\
& \bigcup\{(-1,1,1)\} \cdot \Omega\left(4^{+}, 0\right), \quad \text { Figure 6f } \\
& +z r(c-1) F(r, 0) \\
& +z(c-1)(F(r, 0)-F(0,0)) \\
& +z s(c-1)\left[r^{1}\right] F(r, s) \\
& +z(c-1)\left(\left[r^{1}\right] F(r, s)-\left[r^{1}\right] F(r, 0)\right) \\
& \bigcup\{(1,1,1)\} \cdot \Omega\left(2^{+}, 0\right), \quad \text { Figure } 6 \mathrm{~g} \\
& \bigcup\{(1,-1,-1)\} \cdot \Omega\left(0^{+}, 0\right), \quad \text { Figure } 6 \mathrm{~h} \\
& \bigcup\{(-1,-1,-1)\} \cdot \Omega\left(2^{+}, 0\right), \quad \text { Figure 6i } \\
& +z r(c-1)\left[s^{1}\right] F(r, s) \\
& \bigcup\{(-1,1,-1)\} \cdot \Omega\left(2,0^{+}\right), \quad \text { Figure } 6 \mathrm{j} \\
& \bigcup\{(-1,1,1)\} \cdot \Omega\left(2,2^{+}\right), \quad \text { Figure } 6 \mathrm{k} \\
& \bigcup\{(1,-1,1)\} \cdot \Omega\left(0^{+}, 2\right), \quad \text { Figure 6] }
\end{aligned}
$$




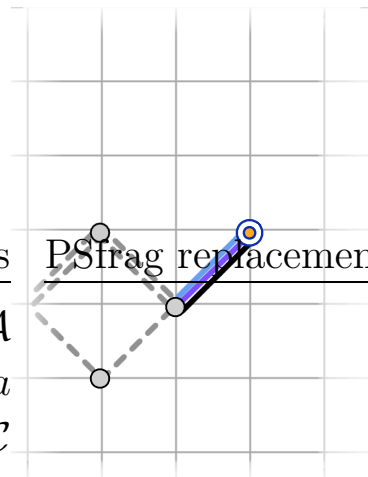

(a)

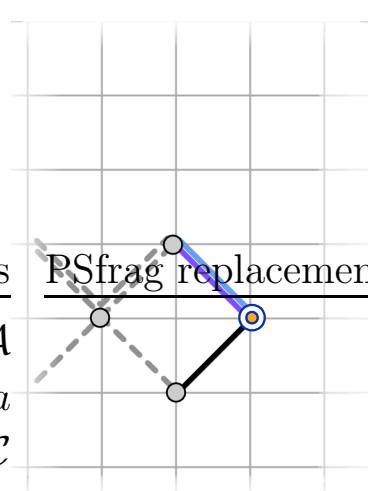

(c)

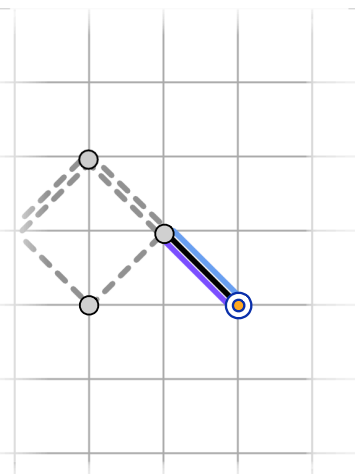

(b)

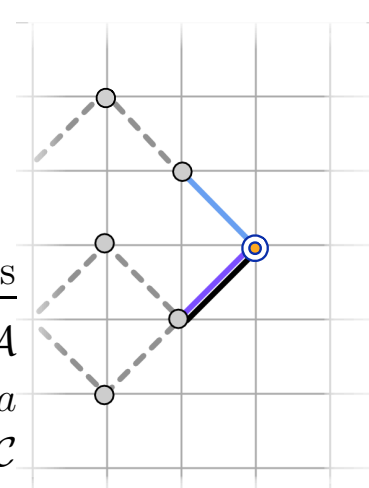

(d)

Figure 5. The four possible ways of appending a triplet of steps to an allowed configuration that results in all walks visiting the same site. 


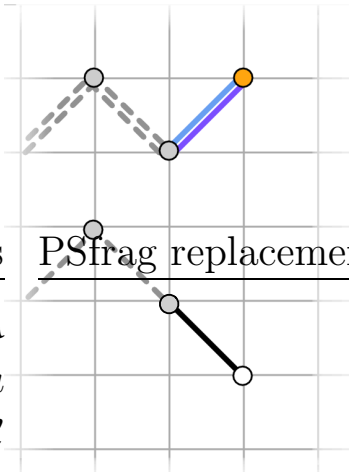

(a)

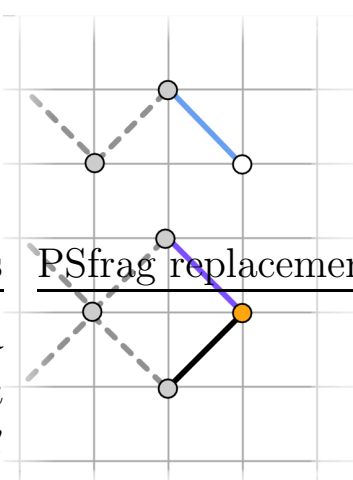

(e)

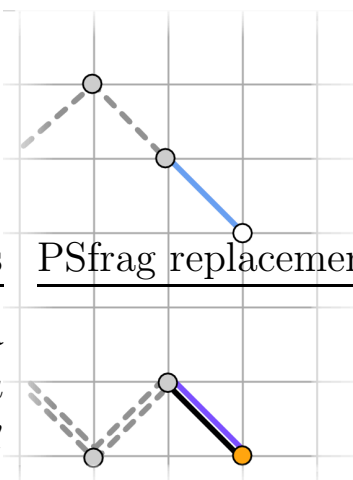

(i)

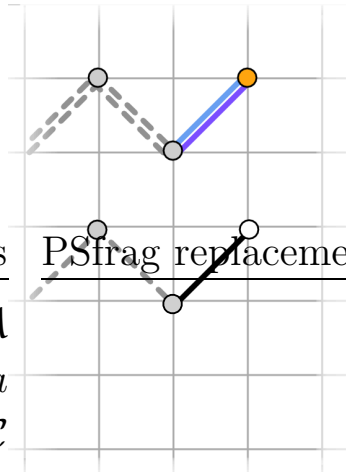

(b)

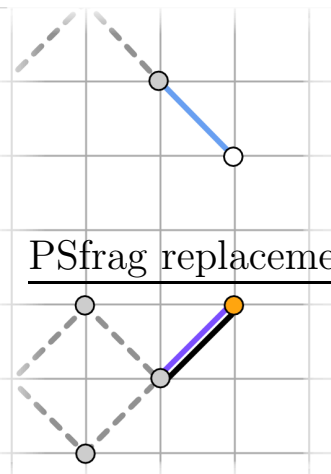

(f)

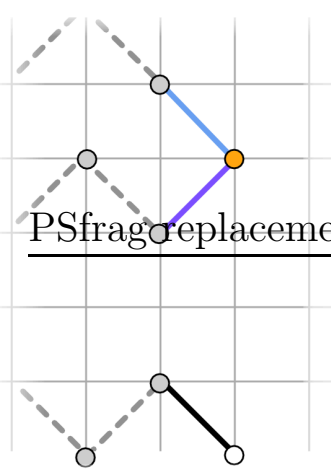

(j)

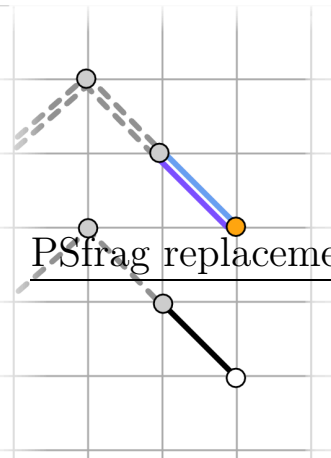

(c)

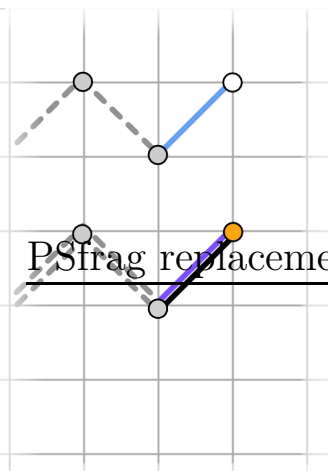

(g)

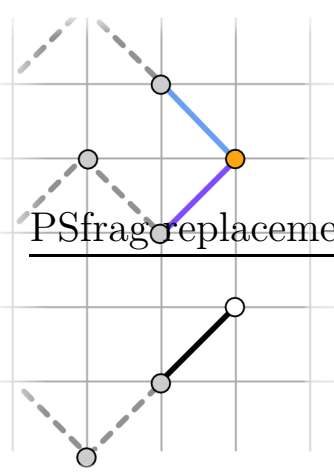

(k)

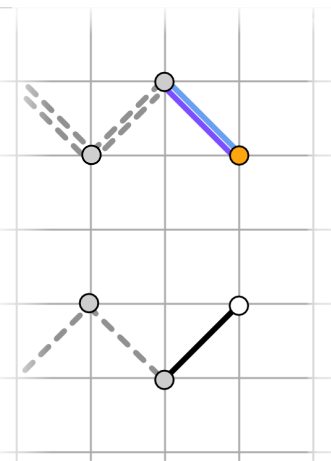

(d)

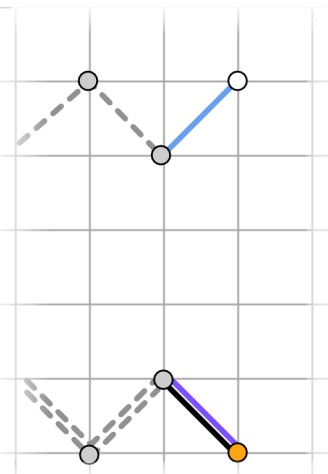

(h)

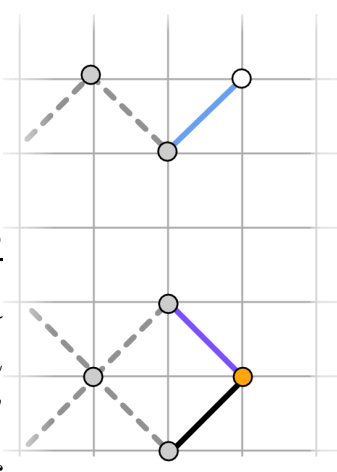

(1)

Figure 6. The twelve possible ways of appending a triplet of steps to an allowed configuration where only two of the three walks visit the same site. 
We now further refine (17) by eliminating the terms $\left[s^{1}\right] F(0, s),\left[r^{1}\right] F(r, 0)$, $\left[s^{1}\right] F(r, s)$ and $\left[r^{1}\right] F(r, s)$. The first step in this process is to construct 3 new functional equations for $F(0,0), F(0, s)$ and $F(r, 0)$ :

$$
\begin{aligned}
& F(0,0)=\quad \Omega(0,0)= \\
& 1 \\
& +z c^{2} F(0,0) \quad \bigcup\{(1,1,1)\} \cdot \Omega(0,0) \quad \text { Figure 5a } \\
& +z c^{2} F(0,0) \quad \bigcup\{(-1,-1,-1)\} \cdot \Omega(0,0) \quad \text { Figure } 5 \mathrm{~b} \\
& +z c^{2}\left[s^{1}\right] F(0, s) \quad \bigcup\{(-1,-1,1)\} \cdot \Omega(0,1) \quad \text { Figure } 5 \mathrm{c} \\
& +z c^{2}\left[r^{1}\right] F(r, 0) \quad \bigcup\{(-1,1,1)\} \cdot \Omega(1,0) \quad \text { Figure } 5 \mathrm{~d} \\
& \text { and } \\
& F(0, s)= \\
& 1 \\
& +z c^{2} F(0,0) \\
& +z c^{2} F(0,0) \\
& +z c^{2}\left[s^{1}\right] F(0, s) \\
& +z c^{2}\left[r^{1}\right] F(r, 0) \\
& +z \operatorname{scF}(0, s) \\
& +z c(F(0, s)-F(0,0)) \\
& +z c(F(0, s)-F(0,0)) \\
& +\frac{z}{s}(c-1)\left(F(0, s)-F(0,0)-s\left[s^{1}\right] F(0, s)\right) \\
& +z s c\left(\left[r^{1}\right] F(r, s)\right) \\
& +z c\left(\left[r^{1}\right] F(r, s)-\left[r^{1}\right] F(r, 0)\right) \\
& \Omega\left(0,0^{+}\right)= \\
& \{\bullet\} \\
& \bigcup\{(1,1,1)\} \cdot \Omega(0,0), \quad \text { Figure 5a } \\
& \bigcup\{(-1,-1,-1)\} \cdot \Omega(0,0), \quad \text { Figure } 5 \mathrm{~b} \\
& \bigcup\{(-1,-1,1)\} \cdot \Omega(0,1) \quad \text { Figure } 5 \mathrm{c} \\
& \bigcup\{(-1,1,1)\} \cdot \Omega(1,0) \quad \text { Figure } 5 \mathrm{~d} \\
& \bigcup\{(1,1,-1)\} \cdot \Omega\left(0,0^{+}\right), \quad \text { Figure 6a } \\
& \bigcup\{(1,1,1)\} \cdot \Omega\left(0,2^{+}\right), \quad \text { Figure 6b } \\
& \bigcup\{-1,-1,-1\} \cdot \Omega\left(0,2^{+}\right) \text {, Figure } 6 \mathrm{c} \\
& \bigcup\{-1,-1,1\} \cdot \Omega\left(0,4^{+}\right), \quad \text { Figure } 6 \mathrm{~d} \\
& \bigcup\{(-1,1,-1)\} \cdot \Omega\left(2,0^{+}\right), \quad \text { Figure 6j } \\
& \bigcup\{(-1,1,1)\} \cdot \Omega\left(2,2^{+}\right), \quad \text { Figure 6k }
\end{aligned}
$$


Three interacting friendly walks

and

$$
\begin{aligned}
& F(r, 0)= \\
& 1 \\
& +z c^{2} F(0,0) \\
& +z c^{2} F(0,0) \\
& +z c^{2}\left[s^{1}\right] F(0, s) \\
& +z c^{2}\left[r^{1}\right] F(r, 0) \\
& +z r c F(r, 0) \\
& +z c(F(r, 0)-F(0,0)) \\
& +z c(F(r, 0)-F(0,0)) \\
& +\frac{z}{r}(c-1)\left(F(r, 0)-F(0,0)-r\left[r^{1}\right] F(r, 0)\right) \\
& +z r c\left(\left[s^{1}\right] F(r, s)\right) \\
& +z c\left(\left[s^{1}\right] F(r, s)-\left[s^{1}\right] F(r, 0)\right)
\end{aligned}
$$

$$
\Omega\left(0^{+}, 0\right)=
$$

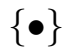

$\bigcup\{(1,1,1)\} \cdot \Omega(0,0), \quad$ Figure 5a

$\bigcup\{(-1,-1,-1)\} \cdot \Omega(0,0), \quad$ Figure $5 \mathrm{~b}$

$\bigcup\{(-1,-1,1)\} \cdot \Omega(0,1) \quad$ Figure $5 \mathrm{c}$

$\bigcup\{(-1,1,1)\} \cdot \Omega(1,0) \quad$ Figure $5 \mathrm{~d}$

$\bigcup\{(-1,-1,1)\} \cdot \Omega\left(0^{+}, 0\right), \quad$ Figure $6 \mathrm{e}$

$\bigcup\{(-1,-1,-1)\} \cdot \Omega\left(2^{+}, 0\right), \quad$ Figure 6i

$\bigcup\{1,1,1\} \cdot \Omega\left(2^{+}, 0\right)$, Figure $6 \mathrm{~g}$

$\bigcup\{1,1,-1\} \cdot \Omega\left(4^{+}, 0\right), \quad$ Figure 6a

$\bigcup\{(1,-1,1)\} \cdot \Omega\left(0^{+}, 2\right), \quad$ Figure 61

$\bigcup\{(1,-1,-1)\} \cdot \Omega\left(2^{+}, 2\right)$, Figure 6h,

Next we solve this system for three of the terms we wish to eliminate (say $\left[s^{1}\right] F(0, s),\left[r^{1}\right] F(r, 0)$ and $\left.\left[s^{1}\right] F(r, s)\right)$, and then subsitute these back into (17). In so doing, we find that the fourth term, $\left[r^{1}\right] F(r, s)$, is also eliminated. This results in an equation satisfied by $F(r, s)$ that is considerably simpler:

$$
\begin{aligned}
K(r, s) F(r, s) & =\frac{1}{c^{2}}-\frac{(r-c r+c z+c s z)}{c r} F(0, s) \\
& -\frac{(s-c s+c z+c r z)}{c s} F(r, 0) \\
& -\frac{(c-1)^{2}}{c^{2}} F(0,0)
\end{aligned}
$$

where the kernel, $K(r, s)$, is

$$
K(r, s) \equiv K(r, s ; z)=1-\frac{z(r+1)(s+1)(r+s)}{r s} .
$$

\subsection{Symmetries and roots of the kernel}

We observe that the kernel $K(r, s)$ in (21) and (22) is symmetric under the two transformations

$$
\Phi:(r, s) \mapsto(s, r), \quad \Psi:(r, s) \mapsto\left(r, \frac{r}{s}\right),
$$

where both $\Phi$ and $\Psi$ are involutions. These transformations generate a family of 12 symmetries $\mathcal{F}$ - namely,

$$
\mathcal{F}=\left\{(r, s),(s, r),\left(r, \frac{r}{s}\right),\left(s, \frac{s}{r}\right),\left(\frac{r}{s}, r\right),\left(\frac{s}{r}, s\right),\left(\frac{r}{s}, \frac{1}{s}\right),\left(\frac{s}{r}, \frac{1}{r}\right),\left(\frac{1}{s}, \frac{r}{s}\right),\left(\frac{1}{r}, \frac{s}{r}\right),\left(\frac{1}{r}, \frac{1}{s}\right),\left(\frac{1}{s}, \frac{1}{r}\right)\right\} .
$$


Now, considering $K(r, s)$ as a polynomial in $r$, we find two roots $\hat{s}_{ \pm}$

$$
\begin{aligned}
& \hat{s}_{ \pm}(r ; z)=\frac{r-z\left(r^{2}+2 r+1\right) \pm \sqrt{r^{2}-2 z r(1+r)^{2}+z^{2}\left(r^{2}-1\right)^{2}}}{2 z(r+1)}, \\
& \hat{s}_{-}(r ; z)=(r+1) z+\frac{(r+1)^{3} z^{2}}{r}+O\left(z^{3}\right), \\
& \hat{s}_{+}(r ; z)=\frac{r}{(r+1) z}-(r+1)+O(z) .
\end{aligned}
$$

Moreover, as the kernel is symmetric in $r$ and $s$, we additionally have the roots $\hat{r}_{ \pm}$

$$
\hat{r}_{ \pm}(s ; z)=\frac{s-z\left(s^{2}+2 s+1\right) \pm \sqrt{s^{2}-2 z s(1+s)^{2}+z^{2}\left(s^{2}-1\right)^{2}}}{2 z(s+1)},
$$

Providing $F\left(r, \hat{s}_{ \pm}\right)$remains in the ring of formal power series $\mathbb{Z}[r, a, c][[z]]$, substituting $s \mapsto \hat{s}_{ \pm}$into (21) sets the kernel to zero and eliminates the left-hand side of the functional equation. Similarly, providing $F\left(\hat{r}_{ \pm}, s\right)$ lies in $\mathbb{Z}[s, a, c][[z]]$, substituting $r \mapsto \hat{r}_{ \pm}$will set the left-hand side of the functional equation to zero.

By considering partial sums of $F(r, s)$ up to $O\left(z^{n}\right)$, one can show that $F\left(r, \hat{s}_{-}\right)$ and $F\left(\hat{r}_{-}, s\right)$ converge within the desired rings, while the other substitutions do not (essentially because $\hat{r}_{+}, \hat{s}_{+}=O\left(z^{-1}\right)$ ). Hence for the remainder of this paper, we define $\hat{s}(r ; z) \equiv \hat{s}_{-}(r ; z)$ and $\hat{r}(s ; z) \equiv \hat{r}_{-}(s ; z)$, and only consider substitution of these roots.

Finally, when the kernel (22) $K(r, s)=0$ we have

$$
r s=z(r+1)(s+1)(r+s)
$$

and thus Lagrange inversion [12] yields

$$
\begin{aligned}
& \hat{r}(s ; z)^{k}=\sum_{n \geq k} \frac{k}{n} z^{n}(1+s)^{n} \sum_{j=k}^{n}\left(\begin{array}{c}
n \\
j
\end{array}\right)\left(\begin{array}{c}
n \\
j-k
\end{array}\right) s^{j-n} \\
& \hat{s}(r ; z)^{k}=\sum_{n \geq k} \frac{k}{n} z^{n}(1+r)^{n} \sum_{j=k}^{n}\left(\begin{array}{c}
n \\
j
\end{array}\right)\left(\begin{array}{c}
n \\
j-k
\end{array}\right) r^{j-n} .
\end{aligned}
$$

Note that the above are closely related to the generating function of the Narayana

numbers $N(n, j)=\frac{1}{n}\left(\begin{array}{c}n \\ j\end{array}\right)\left(\begin{array}{c}n \\ j-1\end{array}\right)$. This observation is sufficient to ensure that the above series contain only non-negative integer coefficients.

These explicit series representations for positive integer powers of the roots $\hat{r}$ and $\hat{s}$, will be used below to help find an explicit expression for the generating function.

\subsection{Using the symmetries of the kernel}

Equipped with the roots $\hat{s}$ and $\hat{r}$ as well as the family of symmetries $\mathcal{F}$ that leave the kernel invariant, we can now apply the obstinate kernel method. Specifically, we substitute $(r, s) \mapsto(r, \hat{s})$ and $(r, s) \mapsto(\hat{r}, s)$ into the simplified functional equation (21), subsequently applying a subset of transformations from $\mathcal{F}$ to generate a system of new functional equations. Mapping $(r, s) \mapsto(\hat{r}, s)$ we have the system

$0=\frac{1}{c^{2}}-\frac{(\hat{r}-c \hat{r}+c z+c s z)}{c \hat{r}} F(0, s)-\frac{(\hat{s}-c s+c z+c \hat{r} z)}{c s} F(\hat{r}, 0)-\frac{(c-1)^{2}}{c^{2}} F(0,0), \quad(r, s) \mapsto(\hat{r}, s)$ 
Three interacting friendly walks

$0=\frac{1}{c^{2}}-\frac{\left(\hat{r}-c \hat{r}+c z+\frac{c \hat{r} z}{s}\right)}{c r} F\left(0, \frac{\hat{r}}{s}\right)-\frac{s\left(\frac{\hat{r}}{s}-\frac{c \hat{r}}{s}+c z+c \hat{r} z\right)}{c \hat{r}} F(\hat{r}, 0)-\frac{(c-1)^{2}}{c^{2}} F(0,0), \quad(r, s) \mapsto\left(\hat{r}, \frac{\hat{r}}{s}\right)$

$0=\frac{1}{c^{2}}-\frac{s\left(\frac{1}{s}-\frac{c}{s}+c z+\frac{c \hat{r} z}{s}\right)}{c} F\left(0, \frac{\hat{r}}{s}\right)-\frac{s\left(\frac{\hat{r}}{s}-\frac{c \hat{r}}{s}+c z+\frac{c z}{s}\right)}{c \hat{r}} F\left(\frac{1}{s}, 0\right)-\frac{(c-1)^{2}}{c} F(0,0), \quad(r, s) \mapsto\left(\frac{1}{s}, \frac{\hat{r}}{s}\right)$

where the chosen subset of transformations guarantee that each functional equation (29) - 31) only contain non-negative powers of $\hat{r}$ and thus the generating functions are formally convergent in $\mathbb{Z}[c, s, \bar{s}][[z]]$. Considering the system of equations (29) - (31), we can eliminate $F(\hat{r}, 0)$ by

$$
\begin{aligned}
0 & =[\text { coeff. of } F(\hat{r}, 0) \text { in }(30)] \times[\text { RHS of (29) }] \\
& -[\text { coeff. of } F(\hat{r}, 0) \text { in (29) }] \times[\text { RHS of (30) }] \\
& =-\frac{s\left(\frac{\hat{r}}{s}-\frac{c \hat{r}}{s}+c z+c \hat{r} z\right)}{c \hat{r}}[\text { RHS of (29) }]+\frac{(s-c s+c z+c \hat{r} z)}{c s}[\text { RHS of (30) }] .
\end{aligned}
$$

In a similar vein we can eliminate $F\left(0, \frac{\hat{r}}{s}\right)$ from the system by

$$
\begin{aligned}
0 & =\left[\operatorname{coeff} . \text { of } F\left(0, \frac{\hat{r}}{s}\right) \text { in (32) }\right] \times[\text { RHS of (29) }] \\
& -\left[\operatorname{coeff.~of~} F\left(0, \frac{\hat{r}}{s}\right) \text { in (29) }\right] \times[\text { RHS of (32)] }] \\
& =\frac{(s-c s+c z+c \hat{r} z)(-\hat{r} s+c \hat{r} s-c \hat{r} z-c s z)}{c \hat{r} s^{2}}[\text { RHS of (29) }] \\
& -\frac{s\left(-\frac{1}{s}+\frac{c}{s}-c z-\frac{c \hat{r} z}{s}\right)}{c}[\text { RHS of (32) }]
\end{aligned}
$$

yielding a functional equation solely in terms of the generating functions $F(0, s), F(1 / s, 0)$ and $F(0,0)$. Specifically, we have

$N_{1}(s, c ; z) F(1 / s, 0)+N_{2}(s, c ; z) F(0, s)+N_{3}(s, c ; z)\left[(c-1)^{2} F(0,0)-1\right]=0$

where

$$
\begin{aligned}
N_{1}(s, c ; z) & =\frac{(s-c s+c(1+\hat{r}) z)(\hat{r}-c \hat{r}+c(1+s) z)(-(-1+c) \hat{r} s+c(\hat{r}+s) z)}{c^{3} \hat{r}^{2} s^{2}}, \\
N_{2}(s, c ; z) & =\frac{(\hat{r}-c \hat{r}+c s z+c \hat{r} s z)((-1+c) \hat{r}-c(1+s) z)(1+c(-1+(\hat{r}+s) z))}{c^{3} \hat{r}^{2}}, \\
N_{3}(s, c ; z) & =\frac{(c-1)^{2}}{c^{4}}-\frac{(1+\hat{r})(\hat{r}+s)\left(-1+\hat{r} s-s^{3}\right) z^{2}}{c^{2} \hat{r} s^{2}} \\
& -\frac{\left(-c \hat{r}+c^{2} \hat{r}-c s+c^{2} s-c s^{2}+c^{2} s^{2}-c \hat{r} s^{2}+c^{2} \hat{r} s^{2}\right) z}{c^{4} \hat{r} s} .
\end{aligned}
$$

By an identical process, we can alternatively substitute in the root $\hat{s}$ along with a subset of transformations in $\mathcal{F}$

$$
(r, s) \mapsto(r, \hat{s}),\left(\frac{\hat{s}}{r}, \hat{s}\right),\left(\frac{\hat{s}}{r}, \frac{1}{r}\right)
$$

that contain positive powers of $\hat{s}$ to yield an alternate refined functional equation containing $F(r, 0), F(0,1 / r)$ and $F(0,0)$. However, under the horizontal reflection

$$
(r, s, c) \mapsto(s, r, c)
$$


the generating $F(r, s)$ along with any corresponding functional equations will be invariant and it thus suffices to solely consider (34) for our subsequent analysis.

By using the symmetries of the kernel we have established the refined functional equation (34) containing unknown generating functions in only the catalytic variable $s$. The potential benefit of this new equation is that by extracting the coefficients of $s^{i}$ for some choice of $i$ we hope to establish a relation solely in terms of $F(0,0, c ; z) \equiv G(c, 1 ; z)$. This is indeed what we will proceed to do in Section 4.

\section{Solving the generating function}

Our aim is to utilise the refined functional equations that was established in Section 3.2 . We start by dividing equation (34) by the coefficient of $F(0, s)$, namely $N_{2}(s, c ; z)$. It was then observed that $N_{1} / N_{2}$ was actually a rational function of $s, c$ and $z$. Multiplying through by the associated denominator then gives:

$$
\begin{aligned}
& s-c s+c z+(-1+c) s^{2}(-1+c+c z) F(1 / s, 0) \\
& -s\left(1+s+c\left(-2+c-s+\left(-1+c+s^{2}\right) z\right)\right) F(0, s) \\
& =-s\left(1+s+c\left(-2+c-s+\left(-1+c+s^{2}\right) z\right)\right) \frac{N_{3}(s, c ; z)}{N_{2}(s, c ; z)}\left[1-(c-1)^{2} F(0,0)\right],
\end{aligned}
$$

where our algebraic functions $N_{i}(s, c ; z)$ are defined in (35). Extracting the coefficient of $s^{1}$ of (38) gives us

$$
\begin{aligned}
& 1-c-(c-1)(c z+c-1) F(0,0)+(c-1)(c z+c-1)\left[s^{1}\right] F(s, 0) \\
& =-\left[s^{1}\right]\left\{s\left(1+s+c\left(-2+c-s+\left(-1+c+s^{2}\right) z\right)\right) \frac{N_{3}(s, c ; z)}{N_{2}(s, c ; z)}\right\}\left[1-(c-1)^{2} F(0,0)\right] .
\end{aligned}
$$

Finally, to eliminate the boundary term $\left[s^{1}\right] F(s, 0)$, we recall relation (18) and observe that our walks are symmetric under a horizontal reflection. Thus, $\left[r^{1}\right] F(r, 0)=$ $\left[s^{1}\right] F(0, s)$ (more generally $\left.F(r, s)=F(s, r)\right)$ and so we have the relation

$$
\left[s^{1}\right] F(s, 0)=-\frac{1}{2 c^{2} z}-\frac{\left(-1+2 c^{2} z\right) F(0,0)}{2 c^{2} z},
$$

which when subsequently substituting into (39) yields an expression for $F(0,0)$

$G(c, 1)=F(0,0)=\frac{1}{(c-1)^{2}}\left(1+\frac{c\left(2-3 c+c^{2}-4 c z+c^{2} z+2 c^{3} z+4 c^{2} z^{2}\right)}{1-c+c\left(-1-4 c+4 c^{2}\right) z-2(c-1) c^{2} z\left[s^{1}\right] H(c ; z)+4 c^{3} z^{2}}\right)$

where

$$
H(c ; z) \equiv-s\left(1+s+c\left(-2+c-s+\left(-1+c+s^{2}\right) z\right)\right) \frac{N_{3}(s, c ; z)}{N_{2}(s, c ; z)} .
$$

What remains is to explicitly extract the coefficient of $s^{1}$ from $H(r, c ; z)$. We begin by expanding $H(c ; z)$ as a power series in $c$ giving us

$$
H(c ; z)=\left[s^{4} z+2 s^{3} z+s^{2}(2 z-1)+s z+z\right] \sum_{m \geq 0} c^{m} X(c ; z)^{m}
$$


where

$$
\begin{aligned}
X(c ; z)^{m} & =\frac{z^{m}(s+1)^{m-1}}{s^{m+1}}[\hat{r}+(1+s)]^{m} \\
& =\frac{z^{m}}{s^{m+1}} \sum_{k=0}^{m}\left(\begin{array}{c}
m \\
k
\end{array}\right) \hat{r}^{k}(s+1)^{2 m-k-1}
\end{aligned}
$$

Using our expansion for $\hat{r}^{k}$ in (28) we find

$$
X(c ; z)^{m}=\sum_{k=0}^{m}\left(\begin{array}{c}
m \\
k
\end{array}\right) \sum_{n \geq k} \frac{k}{n} z^{m+n}(s+1)^{2 m+n-k-1} \sum_{j=k}^{n}\left(\begin{array}{c}
n \\
j
\end{array}\right)\left(\begin{array}{c}
n \\
j-k
\end{array}\right) s^{j-m-n-1} .
$$

Now, from (43),$\left[c^{m} s^{1}\right] H(c ; z)$ is

$$
\left[c^{m} s^{1}\right] H(c ; z)=\left(z\left[s^{-3}\right]+2 z\left[s^{-2}\right]+(2 z-1)\left[s^{-1}\right]+z\left[s^{0}\right]+z\left[s^{1}\right]\right) X(c ; z)^{m} .
$$

Thus, equipped with our expansion for $X(c ; z)^{m}$ (45), one can explicitly find $\left[c^{m} s^{1}\right] H(c ; z)$ by extracting and subsequently combining the coefficients $\left[s^{i}\right] X(c ; z)^{m}$ for $i=-3,-2, \ldots, 1$. For instance, we find $\left[s^{0}\right] X(c ; z)^{m}$

$$
\begin{aligned}
{\left[s^{0}\right] X(c ; z)^{m} } & =\sum_{k=1}^{m}\left(\begin{array}{c}
m \\
k
\end{array}\right) \sum_{n \geq k} \frac{k}{n} z^{m+n} \sum_{j=k}^{n}\left(\begin{array}{c}
2 m+n-k-1 \\
m+n-j+1
\end{array}\right)\left(\begin{array}{c}
n \\
j
\end{array}\right)\left(\begin{array}{c}
n \\
j-k
\end{array}\right) \\
& +z^{m}\left(\begin{array}{c}
2 m-1 \\
m+1
\end{array}\right) .
\end{aligned}
$$

Therefore, along with $\left[s^{0}\right] X(c ; z)^{m}$ found in (47) there remain four other components in (46) whose series representation can be determined in the same fashion, giving us an expansion for $\left[s^{1}\right] H(c ; z)$ as a series in $c$. Finally, we change the order of summation to get terms that are a power series in $z$ and with the aid of Maple [13] to combine our sums we find the exact solution for $\left[s^{1}\right] H(c ; z)$ to be

$$
\begin{aligned}
& {\left[s^{1}\right] H(c ; z)=z+\frac{1-b}{b}+\frac{1-2 c z-c^{2} z+\left(c^{2} z-2 c^{2} z^{2}-1\right) \sqrt{1-4 c z}}{2 c^{2} z \sqrt{1-4 c z}}} \\
& +J(c ; z)
\end{aligned}
$$

where $J(c ; z)$ is

$$
\begin{aligned}
J(c ; z)=\sum_{i \geq 3} z^{i} \sum_{m=1}^{i-1} c^{m} \sum_{k=1}^{i-m-1}\left(\begin{array}{c}
m \\
k
\end{array}\right) \sum_{j=k}^{i-m-1} & \left\{\frac{k}{i-m-1}\left(\begin{array}{c}
i-m-1 \\
j
\end{array}\right)\left(\begin{array}{c}
i-m-1 \\
j-k
\end{array}\right)\left[\left(\begin{array}{c}
m+i-k \\
i-j
\end{array}\right)+\left(\begin{array}{c}
m+i-k \\
i-j-2
\end{array}\right)\right]\right. \\
& \left.-\frac{k}{i-m}\left(\begin{array}{c}
i-m \\
j
\end{array}\right)\left(\begin{array}{c}
i-m \\
j-k
\end{array}\right)\left(\begin{array}{c}
m+i-k-1 \\
i-j-1
\end{array}\right)\right\} \\
& -\sum_{i \geq 2} z^{i} \sum_{m=1}^{i-1} c^{m} \sum_{k=1}^{i-m}\left(\begin{array}{c}
m \\
k
\end{array}\right) \frac{k}{i-m}\left(\begin{array}{c}
i-m \\
i-k-m
\end{array}\right)\left(\begin{array}{c}
m+i-k-1 \\
m-1
\end{array}\right)
\end{aligned}
$$

Substituting (48) into (41) yields

$$
G(c, 1 ; z)=\frac{1}{c(c-2)+1}\left(1+\frac{\left[c\left(2-3 c+c^{2}\right)+z c^{3}\right] \sqrt{1-4 c z}}{G_{b}(c, 1 ; z)}\right)
$$

where

$G_{b}(c, 1 ; z)=(1-c)\left(-1+2 c z+c^{2} z\right)+c z \sqrt{1-4 c z}\left[-1+c-c^{2}+2 c(c-1) J(c ; z)\right]$. 
Finally recalling relation (9) between $G(c, d ; z)$ and $G(c, 1 ; z)$, our solution of the full model is

$$
G(c, d ; z)=\frac{1}{c d(c-2)+1}\left(1+\frac{\left[c d(c-2)(c-1)^{2}+c^{3} d z(c-1)\right] \sqrt{1-4 c z}}{G_{b}(c, d ; z)}\right),
$$

where

$$
\begin{aligned}
G_{b}(c, d ; z) & =1-2 c d+c^{2} d-c(c+2)\left(1-2 c d+c^{2} d\right) z \\
& +\sqrt{1-4 c z}\left[c(2-c)(d-1)+c z\left(1-2 c d+2 c^{2} d-c^{3} d\right)+2 z c^{2}\left(1-2 c d+c^{2} d\right) J(c ; z)\right] .
\end{aligned}
$$

\section{Phase structure and transitions}

\subsection{Singularity structure of $G(c, 1)$}

Recall from Section 4 that our exact solution to $G(c, 1)$ was expressed as

$$
G(c, 1 ; z)=\frac{1}{c(c-2)+1}\left(1+\frac{\left[c\left(2-3 c+c^{2}\right)+z c^{3}\right] \sqrt{1-4 c z}}{G_{b}(c, 1 ; z)}\right)
$$

with the denominator $G_{b}(c, 1 ; z)$ defined in (51). In particular $G_{b}(c, 1 ; z)$ is an expression in terms of $z, c$ and the power series $J(c ; z)$ which itself is defined at (49)). Hence the dominant singularity $z_{s}(c, 1)$ of our generating function is dependent on the dominant singularity $z_{J}(c, 1)$ of $J(c ; z)$ along with any poles that arise from the roots of $G_{b}(c, 1 ; z)$. Now, by an exact approach featured in [14, we employ the technique of differentiating hyperexponential functions under the integral sign to determine a linear homogenous differential equation with polynomial coefficients in $z$ and $c$ that is satisfied by the series. Once again, we utilise the Maple package DETools which implements the so-called 'fast' Zeilberger algorithm applicable to hyperexponential functions [15], to find the linear differential operator $\mathcal{L}$ where

$$
\begin{aligned}
& \mathcal{L}=\left[16384 c^{15}\left(279 c^{6} \ldots+23808\right) z^{26}+\ldots+\left(-10 c^{17}+\ldots+4\right) z^{3}\right]\left(\partial / \partial_{z}\right)^{7} \\
& +\left[23592960 c^{15}\left(279 c^{6} \ldots+23808\right) z^{20}+\ldots+\left(-302400 c^{18}+\ldots-72000 c^{2}\right)\right]\left(\partial / \partial_{z}\right), \\
& \quad \text { satisfying the equation } \\
& \quad \mathcal{L} J(c ; z)=0 .
\end{aligned}
$$

In Appendix A we explicitly write out the leading polynomial coefficient of (55) whose zeroes correspond to the singularities of $J(c ; z)$ which allows us to determine the dominant singularity structure $z_{J}(c, 1)$ of $J(c ; z)$

$$
z_{J}(c, 1)= \begin{cases}z_{b} \equiv 1 / 8, & c \leq 4 / 3 \\ z_{c}(c) \equiv \frac{1-c+\sqrt{c^{2}-c}}{2 c}, & c>4 / 3 .\end{cases}
$$

Thus we find a critical point at $c=4 / 3$ when both the singularities $z_{b}=z_{c}(4 / 3)=1 / 8$ coincide. Note that the above implies that the square-root singularity of $G(c, 1 ; z)$ at $z=1 / 4 c$ is subdominant. Recall further that with our differential equation one can also 
determine the corresponding linear recurrence for the coefficients $J_{n}$ of $J(c ; z)$ and in particular with the assistance of the Maple package Gfun [16] we find

$$
\begin{aligned}
& -6120576000(c-1)^{15}\left(2-10 c+5 c^{2}\right) J_{n+23} \\
& +q_{2}(c, n) J_{n+22}+q_{3}(c, n) J_{n+21}+q_{4}(c, n) J_{n+20}+\ldots \\
& +1966080 c^{15}\left(23808-73632 c+82960 c^{2}-35756 c^{3}-2310 c^{4}+4358 c^{5}+279 c^{6}\right) J_{n}=0,
\end{aligned}
$$

where $q_{i}(c, n) \in \mathbb{Z}[c, n]$, giving us a homogeneous linear recurrence equation of order 23 with polynomial coefficients in $n$. The growth of the coefficients $J_{n}$ of $J(c ; z)$ can be directly determined from recurrence (58) by appealing to the method of Wimp and Zeilberger [17], showing the existence and specific form of a basis set of asymptotic solutions for any given linear recurrence which, in particular, contains rational coefficients (in $n$ ). In this instance, we substitute into (158) the ansatz

$$
J_{n}=b_{0} \mu^{n} n^{\gamma-1}, \quad b_{0} \neq 0
$$

where $\mu, \gamma, b_{0} \in \mathbb{R}$. By collecting dominant powers of $n$ and equating their corresponding coefficients to zero we can solve for $\mu, \gamma$. In doing so we find

$$
J_{n} \equiv J_{n}(c)= \begin{cases}B_{-} 8^{n} n^{-4}, & c<4 / 3, \\ B_{0} 8^{n} n^{-2}, & c=4 / 3, \\ B_{+} z_{c}(c)^{-n} n^{-1 / 2}, & c>4 / 3\end{cases}
$$

and moreover that the singular part of the generating function near the radius of convergence behaves as

$$
J(c ; z) \sim \begin{cases}B_{-}(1-8 z)^{3} \log (1-8 z), & c<4 / 3, \\ B_{0}(1-8 z) \log (1-8 z), & c=4 / 3, \\ B_{+}\left(1-z / z_{c}(c)\right)^{-1 / 2}, & c>4 / 3 .\end{cases}
$$

Finally, we want to determine whether $G(c, 1 ; z)$ exhibits any additional critical points that arise from the smallest real and positive root $z_{p}(c)$ of $G_{b}(c ; z)$. By taking a truncated series approximation of $J(c ; z)$, we can numerically find the roots of $G_{b}(c ; z)$ and estimate $z_{p}(c)$ which we plot in Figure 7, suggesting that $z_{p}(c)<z_{c}(c)$ for all $c>4 / 3$. We know that as $c \rightarrow \infty, G(c, 1 ; z)$ is dominated by those configurations where all three walks coincide for every step so that

$$
G(c, 1 ; z) \sim \frac{1}{1-2 c^{2} z}, \quad c \rightarrow \infty .
$$

Hence, we indeed should expect the pole $z_{p}(c)$ to dominate $z_{c}(c)$ for large $c>4 / 3$. Now, we need to justify that $z_{p}(c)<z_{c}(c)$, for all $c>4 / 3$. If we assume the converse, then there must exist $c^{\star}>4 / 3$ such that $z_{p}\left(c^{\star}\right)=z_{c}\left(c^{\star}\right)$. From (61) , the analytic and singular expansion of $J\left(c^{\star} ; z\right)$ is given as

$$
J\left(c^{\star} ; z\right) \sim A_{+}+B_{+}\left(1-z / z_{c}\left(c^{\star}\right)\right)^{-1 / 2}, \quad z \rightarrow z_{c}\left(c^{\star}\right), A_{+}, B_{+} \neq 0,
$$

and our expansion for $G_{b}\left(c^{\star}, 1 ; z\right)$ becomes

$$
G_{b}\left(c^{\star}, 1 ; z\right) \sim f_{0}\left(c^{\star}\right)+\frac{f_{1}\left(c^{\star}\right) B_{+}}{\sqrt{1-z / z_{c}\left(c^{\star}\right)}}+f_{2}\left(c^{\star}\right) B_{+} \sqrt{1-z / z_{c}\left(c^{\star}\right)}, \quad z \rightarrow z_{c}\left(c^{\star}\right),
$$




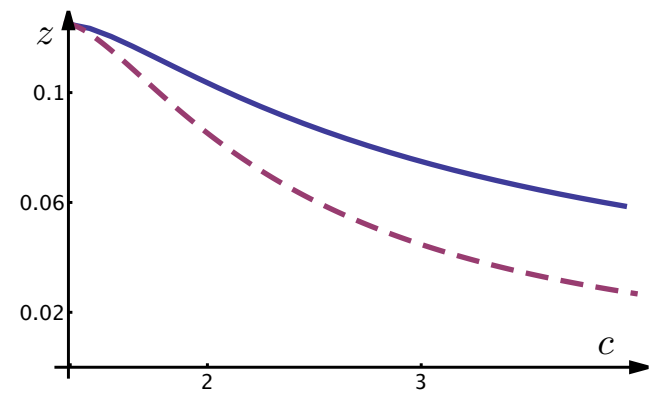

Figure 7. The singularities $z_{p}(c)$ (dashed) and $z_{c}(c)$ (solid) for $c>4 / 3$

where $f_{i}\left(c^{\star}\right)$ are algebraic functions in $c^{\star}$. Note that $A_{+}$has been absorbed into $f_{0}$. Now, as $G_{b}\left(c^{\star}, 1 ; z_{p}\left(c^{\star}\right)\right) \sim 0$, we require $f_{0}=f_{1}=0$, however considering $f_{1}(c)$, where

$$
f_{1}(c)=\frac{2 c^{2}(c-1)^{2}(2-3 c+3 \sqrt{c(c-1)})}{\sqrt{-1+2 c-2 \sqrt{c(c-1)}}},
$$

we find the only roots of $f_{1}$ are at $c=0,1$ and $4 / 3$. Thus, there can not exist $c^{\star}>4 / 3$ such that $z_{c}\left(c^{\star}\right)=z_{p}\left(c^{\star}\right)$. With that in mind, we can finally conclude that the dominant singularity, $z_{s}(c, 1)$, of $G(c, 1 ; z)$ is

$$
z_{s}(c, 1)=\left\{\begin{array}{ll}
z_{b} \equiv 1 / 8, & c \leq 4 / 3, \\
z_{p}(c), & c>4 / 3
\end{array} .\right.
$$

\subsection{Phase transitions of $G(c, 1)$}

Since the dominant singularity of $G(c, 1)$ contains a single non-analytic point, we would expect that our model exhibits two distinct phases. To begin to characterise these phases we introduce the order parameter $\mathcal{C}(c)$ denoting the limiting average number of shared contact sites:

$$
\mathcal{C}(c)=\lim _{L \rightarrow \infty} \frac{\left\langle m_{c}\right\rangle}{L}=c \frac{\partial}{\partial c} \log z_{s}(c, 1) .
$$

The system is in a free phase when

$$
\mathcal{C}=0
$$

while a gelated phase is observed when

$$
\mathcal{C}>0 \text {. }
$$

Recall from section 5.1 that $z_{s}(c, 1)=z_{b} \equiv 1 / 8$ for $c \leq 4 / 3$, implying that $\mathcal{C}=0$ over the same region. For $c \geq 4 / 3, z_{s}(c, 1)=z_{p}(c, 1)$, which is given implicitly as the smallest positive root of the expression

$G_{b}(c, 1 ; z)=-1-c^{2} z-c^{3} z+c(2 z+1)+\sqrt{1-4 c z}\left[-c z+c^{2} z-c^{3} z+\left(-2 c^{2} z+2 c^{3} z\right) J(c ; z)\right]$. 
Now, consider the expansion of $G_{b}\left(c, 1 ; z_{p}(c)\right)=0$ around $c=4 / 3$. With the aid of the Maple [13], we explicitly compute $J(4 / 3 ; 1 / 8)$ where

$$
J(4 / 3 ; 1 / 8)=\frac{-24+13 \sqrt{3}}{8 \sqrt{3}} \approx-0.107051,
$$

and thus as our expansion of $J\left(c ; z_{p}\right)$ is given as

$$
J\left(c ; z_{p}\right) \sim \frac{-24+13 \sqrt{3}}{8 \sqrt{3}}+B_{0}\left(1-8 z_{p}\right) \log \left(1-8 z_{p}\right), \quad c \downarrow 4 / 3,
$$

so that

$G_{b}\left(c, 1 ; z_{p}\right) \sim g_{0}(c-4 / 3)+\left[g_{1}+g_{2}(c-4 / 3)\right]\left(1-8 z_{p}\right) \log \left(1-8 z_{p}\right), \quad c \downarrow 4 / 3, g_{i} \neq 0 .(73)$

As $G_{b}\left(c, 1 ; z_{p}\right) \sim 0$, we apply the implicit function theorem to solve for the dominant behaviour of $\partial z_{p} / \partial c$ in (173) to find

$$
\begin{aligned}
\frac{\partial}{\partial c} z_{p}(c, 1) & \sim \frac{3\left(45-22 \sqrt{3}+72 B\left(1-8 z_{p}\right) \log \left(1-8 z_{p}\right)\right)}{64 B(-32+27 c)\left(1+\log \left(1-8 z_{p}\right)\right)}, \\
& \sim 0, \quad c \downarrow 4 / 3
\end{aligned}
$$

Hence we deduce that our order parameter $\mathcal{C}(c)$ is continuous at $c=4 / 3$. In particular, we find the dominant behaviour second-order derivative of $z_{p}$ is given as

$$
\frac{\partial^{2}}{\partial c^{2}} z_{p}(c, 1) \sim \frac{81(-45+22 \sqrt{3})}{1024 B}, \quad c \downarrow 4 / 3
$$

and we conclude that we observe a second-order phase transition with a finite-jump discontinuity in the first-derivative of $\mathcal{C}(c)$ as seen in Figure 8 . Note, that as $c \rightarrow \infty$, free energy is minimised for those configurations where all three walks are zipped together and the total weight of such a configuration of length $N$ will be $c^{2 N}$. Thus $\mathcal{C}(c) \rightarrow 2$ as $c \rightarrow \infty$ which is indeed what we observe in Figure 8 .

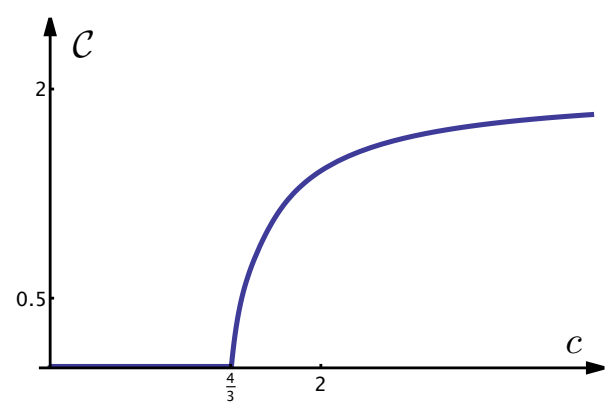

Figure 8. The limiting average number of shared contacts $\mathcal{C}$ when $d=1$. The system exhibits a second-order transition at $c=4 / 3$ respectively 


\subsection{Analysis and phase diagram of the full model}

From our analysis in section 5.1, the dominant singularity $z_{s}(c, d)$ of the generating function can be one of either $z_{b} \equiv 1 / 8$ or the pole $z_{p}(c, d)$ which is now a function of both $c$ and $d$. Substituting $z_{b}$ and $c=4 / 3$ to locate the zero of $G_{b}\left(4 / 3, d ; z_{b}\right)$, we find that the two singularities coincide when $d=9 / 8$ and further that $z_{p}(4 / 3, d)$ is a strictly decreasing function of $d$ for all $d>9 / 8$.

What remains is to determine the dominant singularity over the region $c<4 / 3, d>$ 0 . Overall, estimating the location of $z_{p}(c, d)$ over this region we find

$$
z_{s}(c, d)= \begin{cases}z_{b} \equiv 1 / 8, & c \leq 4 / 3, d<9 / 8 \\ z_{b}, & c \leq \alpha(d), d \geq 9 / 8 \\ z_{p}(c, d), & c>4 / 3, d<9 / 8 \\ z_{p}(c, d), & c>\alpha(d), d \geq 9 / 8\end{cases}
$$

where the boundary $\alpha(d)$ corresponds to when the singularities $z_{p}(c, d)=z_{b}$ coincide respectively. With the full dominant singularity structure established, our system exhibits the same phase regions as per the $d=1$ model - namely, a free phase is observed when

$$
\mathcal{C}=0
$$

while our system is in a gelated phase when

$$
\mathcal{C}>0 \text {. }
$$

Equipped with the phases of our system we plot the phase diagram in Figure 9. By a

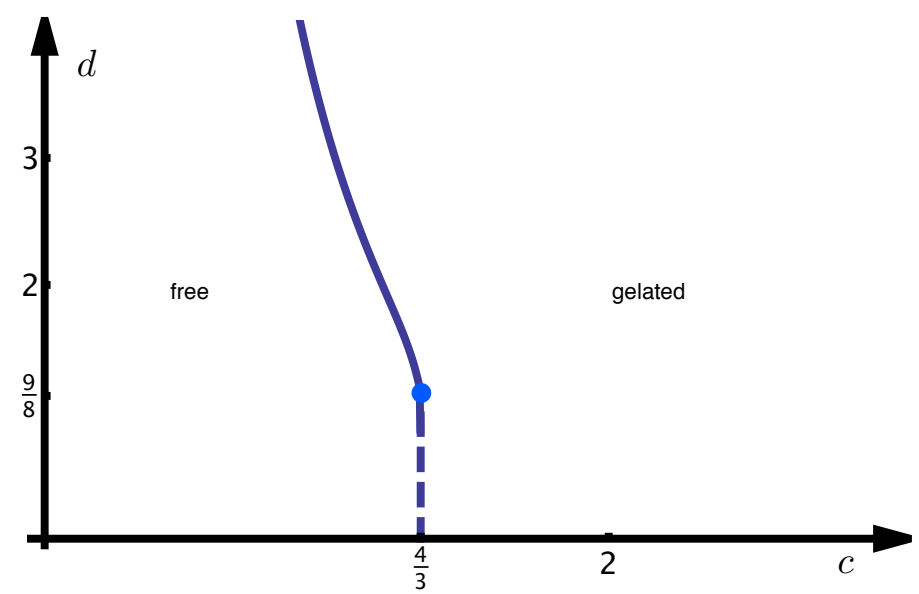

Figure 9. The phase diagram of our full model. First and second-order transitions are indicated by solid and dashed lines respectively. All phase boundaries coincide at $c=4 / 3$ and $d=9 / 8$.

similar argument employed in section 5.2, for general $d<9 / 8$ we observe a second-order transition when moving from a free to gelated phase. Now, considering the boundary 
$\alpha(d)$ where $d>9 / 8$, we note that our our boundary lies in the region $c<4 / 3$, and hence the expansion of $J\left(c ; z_{p}\right)$ is given as

$$
J\left(c ; z_{p}\right) \sim A_{-}+B_{-}\left(1-8 z_{p}\right)^{3} \log \left(1-8 z_{p}\right), \quad c \rightarrow \alpha(d) .
$$

Substituting (79) into $G_{b}\left(c, d ; z_{p}\right)=0$, we again apply the implicit function theorem to solve for the dominant behaviour of $\partial z_{p}(c ; d) / \partial c$ as $c \downarrow \alpha(d)$ to find

$$
\begin{aligned}
& \frac{\partial}{\partial c} z_{p}(c, d) \sim-\frac{17}{8 \alpha}-\frac{15 A_{-}}{4}+\frac{3}{8}(-7+10 d) \\
& +\left(-\frac{15 A_{-}^{2}}{2}+\frac{1}{16} A_{-}(-203+352 d)+\frac{1}{16}\left(-77+122 d-120 d^{2}\right)\right) \alpha+O\left(\alpha^{2}\right), \quad c \rightarrow \alpha(d) .
\end{aligned}
$$

Relying on our estimates for $A_{-}$along $\alpha(d)$, we find that $\partial z_{p}(c ; d) / \partial c$ is non-zero and hence deduce that our order parameter $\mathcal{C}>0$ as $c \downarrow \alpha(d)$. Thus, for $d>9 / 8$, the system exhibits a first-order transition when moving from a free to gelated phase. Moreover, employing a low-temperature argument, we can determine asymptotics for the boundary $\alpha(d)$. Specifically, as $d \rightarrow \infty, G(c, d ; z)$ is dominated by those configurations where all three walks coincide for every step - that is,

$$
G(c, d ; z) \sim \frac{1}{1-2 c^{2} d z}, d \rightarrow \infty,
$$

and equating the singularity of the limiting generating function with $z_{b}$, we find

$$
\alpha(d) \sim 0, \quad d \rightarrow \infty .
$$

Finally, to describe the singular behaviour of $G(c, d)$ across all phases and boundaries, we consider the expansion of the generating function around the dominant singularity $z_{s}(c, d)$. In particular, the singular behaviour of $G(c, d)$ is driven by the expression $G_{b}(c, d)$, and hence $J(c, d)$. Recall in section 5.1 that the singular behaviour of $J(c, d)$ was determined in (60). With that in mind, we find that for the free phase

$$
G_{b}(c, d ; z) \sim f_{0}(c, d)+f_{1}(c, d)(1-8 z)^{3} \log (1-8 z), \quad f_{i} \neq 0,
$$

and thus,

$$
G(c, d ; z)_{\text {singular }} \sim D_{-}(1-8 z)^{3} \log (1-8 z), \quad z \rightarrow \frac{1}{8}, D_{-} \neq 0 .
$$

In a similar fashion, in the gelated phase where $z_{p}(c, d)$ is dominant, $J\left(c, d ; z_{p}\right)$ is analytic and hence

$$
G(c, d ; z)_{\text {singular }} \sim D_{+}\left(1-z / z_{p}(c, d)\right)^{-1}, \quad z \rightarrow z_{p}(c, d), D_{+} \neq 0 .
$$

Now, considering the singular behaviour of $G(c, d)$ along the phase boundaries requires a bit more care. Fixing $c=4 / 3$, we recall from our analysis in section 5.2 that

$$
J(4 / 3 ; 1 / 8)=\frac{-24+13 \sqrt{3}}{8 \sqrt{3}},
$$

implying that

$$
J(4 / 3 ; z) \sim \frac{-24+13 \sqrt{3}}{8 \sqrt{3}}+B_{0}(1-8 z) \log (1-8 z), \quad z \rightarrow \frac{1}{8} .
$$


Three interacting friendly walks

Table 1. The growth rates of the coefficients $Z_{n}(c, d)$ modulo the amplitudes of the full generating function $G(c, d ; z)$ over the entire phase space.

\begin{tabular}{l|c} 
phase region & $Z_{n}(c, d) \sim$ \\
\hline free & $8^{n} n^{-4}$ \\
gelated & $z_{p}(c, d)^{-n} n^{0}$ \\
\hline free to gelated boundary, $d<9 / 8$ & $8^{n} n^{-1} \log n$ \\
free to gelated boundary, $d>9 / 8$ & $8^{n} n^{0}$ \\
\hline$c=4 / 3, d=9 / 8$ & $8^{n} n^{-1}$ \\
\hline
\end{tabular}

Along the free to gelated boundary where $c=4 / 3, d<9 / 8$, we substitute our expansion for $J(4 / 3 ; z)$ in (87) into the generating function and find

$$
\begin{aligned}
G(4 / 3, d ; z)_{\text {singular }} & \sim \frac{-18 d(1-8 z)}{(9-8 d)^{2}\left(24-13 \sqrt{3}+8 \sqrt{3}\left[\frac{-24+13 \sqrt{3}}{8 \sqrt{3}}+B_{0}(1-8 z) \log 1-8 z\right]\right)} \\
& \sim-\frac{18 d}{B_{0}(9-8 d)^{2} \log (1-8 z)}, \quad z \rightarrow \frac{1}{8} .
\end{aligned}
$$

At the point $c=4 / 3, d=9 / 8$, where the two singularities $z_{b}$ and $z_{p}(4 / 3,9 / 8)$ coincide, we have the distinct expression for $G(4 / 3,9 / 8 ; z)$ that arises from a simplification in the generating function $G(4 / 3,1 ; z)$ and hence our primitive generating function $P(4 / 3 ; z)$, where

$$
G(4 / 3,9 / 8 ; z)=\frac{32 z J(4 / 3 ; z)-8+\frac{9}{\sqrt{1-\frac{16 z}{3}}}-4 z\left(-3+\frac{10}{\sqrt{1-\frac{16 z}{3}}}\right)}{1-8 z},
$$

and further we find that the singular behaviour of our generating function at the critical point is

$$
G(4 / 3,9 / 8 ; z)_{\text {singular }} \sim D_{\star} \log (1-8 z), \quad z \rightarrow \frac{1}{8}, D_{\star} \neq 0 .
$$

Finally, considering the boundary $\alpha(d)$, we return to our original expression for the full generating function in (52) where $G_{b} \sim 0$ and along $\alpha(d)$ we find

$$
\begin{aligned}
G(\alpha(d), d ; z)_{\text {singular }} & \sim \frac{D_{\alpha}}{(1-8 z)\left[f_{0}+f_{1}(1-8 z)^{2} \log (1-8 z)\right]}, \quad D_{\alpha}, f_{i} \neq 0 \\
& \sim \frac{D_{\alpha}}{(1-8 z)}, \quad z \rightarrow \frac{1}{8} .
\end{aligned}
$$

Once equipped with the singular behaviour of $G(c, d ; z)$, we can readily obtain the growth rate of the coefficients $Z_{n} \equiv Z_{n}(c, d)$ along the entire phase space, which we summarize in table 1.

\subsection{A change of parameters: $t \equiv d c^{2}$}

Recall from our original model that for a given configuration when all three walks coincide on a site we incorporate both double and triple shared effects, weighting 
that site by $c^{2} d$. As a reparametrization of this model, we can define the parameter $t \equiv c^{2} d$, thereby isolating the effects of double and triple shared contacts which now have corresponding weights $c$ and $t$ respectively. This allows us to consider a system of three interacting polymers where the energy required to graft all three or just two polymers are independent. From (9) we can immediately express the generating function $G(c, t ; z)$ as

$$
G(c, t ; z)=\frac{G(c, 1 ; z)}{\frac{t}{c^{2}}[1-G(c, 1 ; z)]+G(c, 1 ; z)}
$$

where the exact solution to $G(c, 1 ; z)$ has been previously established in (50). Thus the singularity structure of our model (76) remains unchanged except for a rescaling of the pole $z_{p}$ and all singularities coincide at $c=4 / 3, t=2$. More generally the full phase diagram is presented in Figure 10. At $c=0$, the series $J(0 ; z)=1$ and we find the

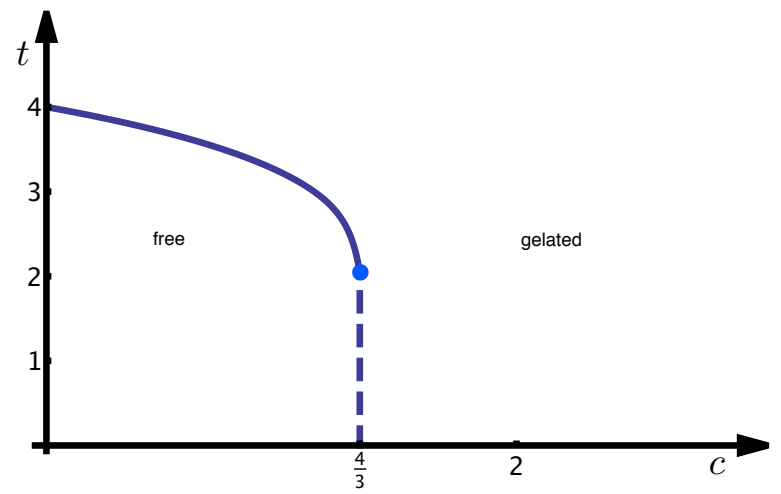

Figure 10. The phase diagram of our full model when setting $d=t / c^{2}$. First and second-order transitions are indicated by solid and dashed lines respectively. All phase boundaries coincide at $c=4 / 3$ and $t=2$.

denominator $G_{b}(0, t ; 1 / 8)=1-t / 4$. Thus we have a critical point at $t=4$, which is precisely what we observe in Figure 10,

\section{Conclusion}

We have solved a model of three interacting friendly directed walks in the bulk. The system has two distinct interaction parameters $c$ and $d$, corresponding to double and triple shared-contact sites to capture the effects of gelation. We established a combinatorial decomposition for the model's full generating function in terms of the corresponding simplified generating function (when $d=1$ ). We then derived a functional equation for this simpler generating function, and by means of the obstinate kernel method, proceeded to solve for both $G(c, 1 ; z)$, and subsequently, the full generating function $G(c, d ; z)$.

Our analysis of the simplified generating function where $d=1$ showed the existence of two phases which we classified as free and gelated, exhibiting a second-order phase transition. We then analysed the full model, presenting the phase diagram and showing 
that the phase space remains partitioned into two distinct phases. In particular, we located second and first-order phase boundaries, which coincide at $c=4 / 3, d=9 / 8$.

It may be natural to consider next a more general asymmetric model incorporating separate parameters for top to middle and middle to bottom shared contacts. We have attempted to analyse this more general model using the same machinery, but unfortunately we have not succeeded in establishing the solution. This may be because the symmetry broken by distinct interactions means that there are insufficient kernel equations to find a full solution. This provides us with an opportunity to explore the limits of application of the obstinate kernel method.

\section{Acknowledgments}

Financial support from the Australian Research Council via its Discovery Projects scheme and the Centre of Excellence for Mathematics and Statistics of Complex Systems is gratefully acknowledged by RT and AO. Financial support from the Natural Sciences and Engineering Research Council of Canada via its Discovery Grant is gratefully acknowledged by AR. RT, acknowledges financial support from the University of Melbourne via its Melbourne Research Scholarships scheme. Additionally, RT and AO thank the Department of Mathematics, University of British Columbia, for hospitality. 


\section{Appendix A. $J(c, z)$ : Leading coefficient of the differential equation}

The following is the leading polynomial coefficient of the linear homogeneous differential equation (55) satisfied by the generating function $J(c ; z)$.

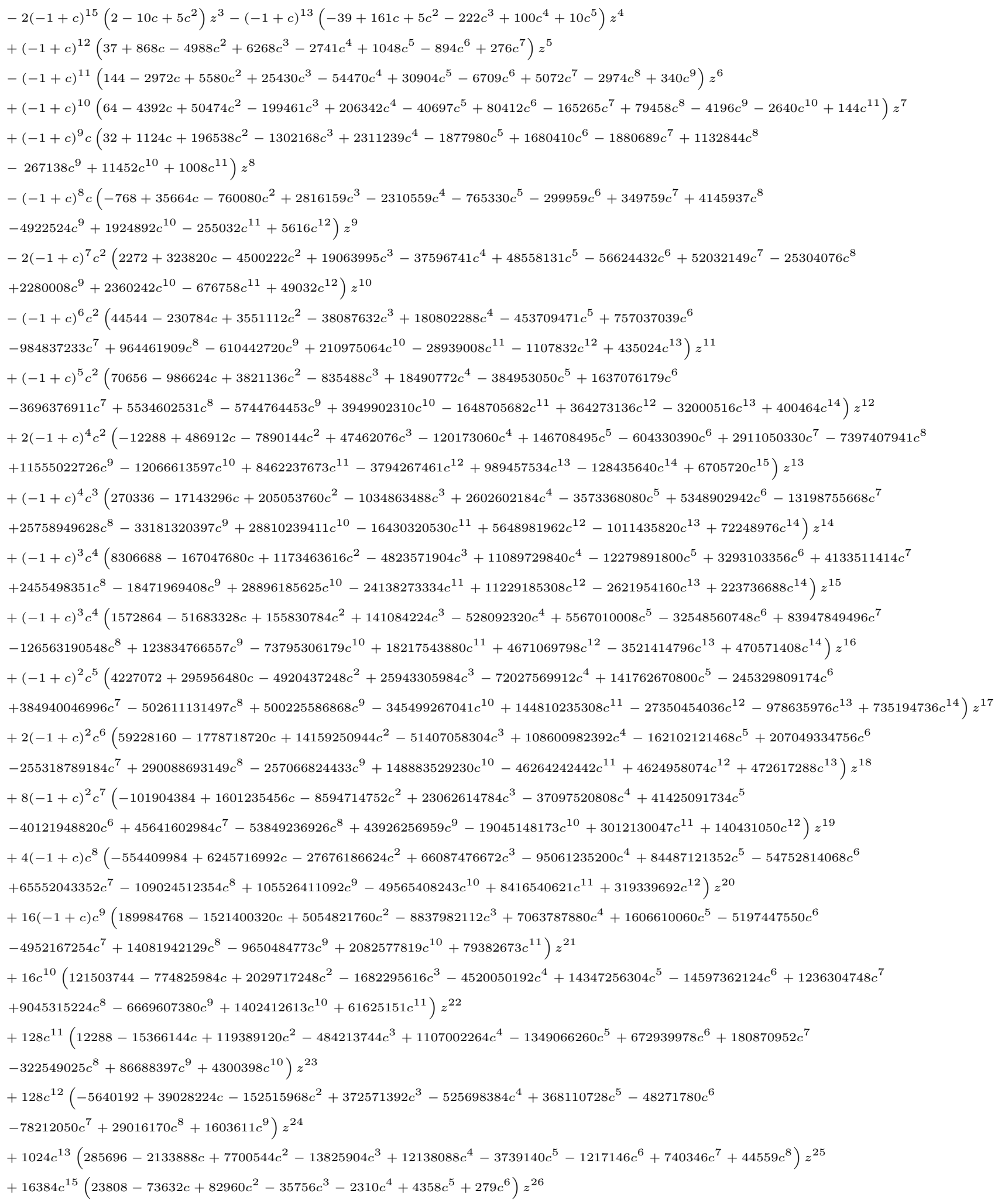




\section{References}

[1] H. G. Elias. Polycondensation and Polyaddition, pages 431-509. Wiley-VCH, 2005.

[2] B. Essevaz-Roulet, U. Bockelmann, and F. Heslot. Mechanical separation of the complementary strands of DNA. Proceedings of the National Academy of Sciences, 94(22):11935, 1997.

[3] D. K. Lubensky and D. R. Nelson. Pulling pinned polymers and unzipping DNA. Physical review letters, 85(7):1572-1575, 2000.

[4] D. K. Lubensky and D. R. Nelson. Single molecule statistics and the polynucleotide unzipping transition. Phys. Rev. E, 65:031917, 2002.

[5] E. Orlandini, S. M. Bhattacharjee, D. Marenduzzo, A. Maritan, and F. Seno. Mechanical denaturation of DNA: existence of a low-temperature denaturation. J. Phys. A, 34:L751, 2001.

[6] D. Marenduzzo, S. M. Bhattacharjee, A. Maritan, E. Orlandini, and F. Seno. Dynamical scaling of the DNA unzipping transition. Physical review letters, 88(2):028102_1-028102_6, 2001.

[7] D. Marenduzzo, A. Maritan, A. Rosa, and F. Seno. Stretching of a polymer below the $\theta$ point. Physical review letters, 90(8):88301, 2003.

[8] D. Marenduzzo, A. Maritan, A. Rosa, F. Seno, and A. Trovato. Phase diagrams for dna denaturation under stretching forces. J. Stat. Mech.: Theor. Exp., 2009(04):L04001, 2009.

[9] A. L. Owczarek, A. Rechnitzer, and T. Wong. Exact solution of two friendly walks above a sticky wall with single and double interactions. Journal of Physics A: Mathematical and Theoretical, 45(42):425003, 2012.

[10] R. Tabbara, A. L. Owczarek, and A Rechnitzer. An exact solution of two friendly interacting directed walks near a sticky wall. Journal of Physics A: Mathematical and Theoretical, 47(1):015202, 2014.

[11] M. Bousquet-Mélou. Counting walks in the quarter plane. In Mathematics and Computer Science II, pages 49-67. Springer, 2002.

[12] P. Flajolet and R. Sedgewick. Analytic combinatorics. cambridge University press, 2009.

[13] Maplesoft: a division of Waterloo Maple Inc. Maple 14 [software package].

[14] R. Tabbara and A. L. Owczarek. Pulling a polymer with anisotropic stiffness near a sticky wall. Journal of Physics A: Mathematical and Theoretical, 45(43):435002, 2012.

[15] G. Almkvist and D. Zeilberger. The method of differentiating under the integral sign. Journal of Symbolic Computation, 10(6):571-591, 1990.

[16] B. Salvy and P. Zimmermann. Gfun: a Maple package for the manipulation of generating and holonomic functions in one variable. ACM Transactions on Mathematical Software, 20(2):163$177,1994$.

[17] J. Wimp and D. Zeilberger. Resurrecting the asymptotics of linear recurrences. Journal of mathematical analysis and applications, 111(1):162-176, 1985. 